\title{
Utilização da glutamina no tratamento de lesões de isquemia e reperfusão no jejuno em eqüinos ${ }^{1}$
}

\author{
Geane Maciel Pagliosa ${ }^{2 *}$, Geraldo Eleno Silveira Alves ${ }^{3}$, Rafael Resende \\ Faleiros $^{3}$, Jorge Rio Tinto de Matos ${ }^{4}$, Márcia Bersane Torres ${ }^{5}$, Heloísa Maria \\ Falcão Mendes ${ }^{6}$ e Renata Sampaio Costa ${ }^{7}$
}

\begin{abstract}
Pagliosa G.M., Alves G.E.S., Faleiros R.R., Matos J.R.T., Torres M.B., Mendes H.M.F. \& Costa R.S. 2009. [Use of glutamine to treatment of ischemia and reperfusion lesions in equine jejune.] Utilização da glutamina no tratamento de lesões de isquemia e reperfusão no jejuno em eqüinos. Pesquisa Veterinária Brasileira 29(3):246-252. Departamento de Clínica e Cirurgia Veterinárias, Escola de Veterinária, Universidade Federal de Minas Gerais, Avenida Antônio Carlos 6627, Caixa Postal 567, Belo Horizonte, MG 30123-970, Brazil. E-mail: geanepagliosa@gmail.com

Under general anesthesia and arterial pressure control, twelve horses were submitted simultaneously to intraluminal distension and venous ischemia of jejune for 2 hours, followed by 12 hours of reperfusion. Six horses were treated with intravenous $2 \%$ glutamine $(50 \mathrm{mg} / \mathrm{kg})$, and six others were treated with the same volume of saline solution $1 \mathrm{~h}$ after the beginning of ischemia and 6 hours after reperfusion. Intestinal samples were collected before and after 2 hours of ischemia and after 2 and 12 hours of reperfusion, and were submitted to hystopatological analyses, ultrastructural examination, measurement of area and number of villi per $\mathrm{mm}^{2}$, and to myeloperoxidases activity. No statistical differences between the groups were found. It was concluded that glutamine did not attenuate the ischemia and reperfusion lesions under the methodology used in this study.
\end{abstract}

INDEX TERMS: Intestine, ischemia and reperfusion, colic, glutamine, equine.

RESUMO.- Sob anestesia geral e com controle da pressão arterial, 12 eqüinos foram submetidos simultaneamente a modelos de isquemia venosa e distensão intraluminal em segmentos do jejuno por $2 \mathrm{~h}$, seguidos de $12 \mathrm{~h}$ de re-

\footnotetext{
${ }^{1}$ Recebido em 11 de agosto de 2008.

Aceito para publicação em 22 de outubro de 2008.

2 Doutoranda em Clínica e Cirurgia Veterinárias, Escola de Veterinária, Universidade Federal de Minas Gerais (UFMG), Belo Horizonte, MG, Brasil. Endereço particular: Rua Milão 1333, Bloco C, Apto 302, Bairro Jardim Itália, Palotina, PR 85950-000, Brasil. *Autor para correspondência: geanepagliosa@gmail.com

${ }^{3}$ Departamento de Clínica e Cirurgia Veterinárias, Escola de Veterinária, UFMG, Av. Antônio Carlos 6627, Cx. Postal 567, Belo Horizonte, MG 30123-970, Brasil.

${ }^{4}$ Centro de Estudos em Clínica e Cirurgia de Animais, Pontifícia Universidade Católica (PUC), Rua do Rosário 1600, Betim, MG 32630000, Brasil.

${ }^{5}$ Curso de Medicina Veterinária, Universidade Federal do Paraná (UFPR), Campus Palotina, Rua Pioneiro 2153, Bairro Jardim Dallas, Palotina PR 85950-000.

${ }^{6}$ Mestranda em Clínica e Cirurgia Veterinárias, Escola de Veterinária, UFMG, Belo Horizonte, MG.

${ }^{7}$ Graduanda do Curso de Medicina Veterinária, Escola de Veterinária, UFMG, Belo Horizonte, MG.
}

perfusão. Seis eqüinos foram tratados com glutamina a $2 \%(50 \mathrm{mg} / \mathrm{kg})$ por via intravenosa e seis com solução fisiológica em igual volume, $1 \mathrm{~h}$ após o início da isquemia e às $6 \mathrm{~h}$ de reperfusão. Foram colhidas amostras de tecido intestinal antes e com $2 \mathrm{~h}$ de isquemia e com 2 e $12 \mathrm{~h}$ de reperfusão para avaliações histopatológica e ultraestrutural, mensuração da área e número das vilosidades por $\mathrm{mm}^{2}$ e atividade de mieloperoxidade (MPO). Não foram encontradas diferenças entre os grupos controle e tratado. Concluiu-se que a glutamina, nas condições deste trabalho, não atenua lesões de isquemia e reperfusão intestinal em eqüinos

TERMOS DE INDEXAÇÃO: intestino, isquemia, reperfusão, cólica, glutamina, eqüino.

\section{INTRODUÇÃO}

A síndrome cólica é uma das afecções de maior prevalência em eqüinos podendo acarretar prejuízos significativos devido ao custo elevado do tratamento e a incidência alta de óbitos. As cólicas podem ser causadas por vários mecanismos, sendo as obstruções intestinais responsáveis por $57 \%$ da ocorrência, com taxas de morte de 
21 e $76 \%$, quando simples ou estrangulantes, respectivamente (White 1990). As obstruções comprometem a circulação intestinal podendo acarretar alterações teciduais que se perpetuam durante a reperfusão, mesmo em segmentos distantes à injúria (Rio Tinto 1999). Nas situações que demandam tratamento cirúrgico é também necessário um aporte terapêutico adequado para minimizar ou atenuar as alterações teciduais.

A glutamina é um aminoácido não essencial presente em altas concentrações no sangue e nas células musculares e intestinais, sendo a principal fonte energética para os enterócitos (Lopes-Paulo 2005, Thomas \& Balasubramanian 2005). A glutamina também participa da proliferação e atividade de fibroblastos e linfócitos e modula a resposta de células intestinais no estresse oxidativo e na inflamação, além de inibir a apoptose induzida pelo TNF-ó em enterócitos (Fuchs \& Bode 2006). Em pacientes portadores de afecções graves, a concentração de glutamina na circulação decresce devido ao aumento da demanda desse nutriente pelos tecidos. Nesses pacientes, a diminuição da glutamina possui uma correlação positiva com a mortalidade, sendo sua suplementação benéfica, o que a torna um aminoácido essencial nesses casos (LopesPaulo, 2005).

A suplementação com glutamina foi benéfica na regeneração da mucosa intestinal e na cicatrização cutânea em humanos portadores de queimaduras e, em condições experimentais, em casos de injúria e reperfusão intestinal, hepática e periférica em ratos (Harward et al. 1994, Peng et al. 2004, Jia et al. 2006, Murphy et al. 2007, Szijártó et al. 2007). A glutamina foi indicada em casos de transplantes ósseos e cirurgias intestinais em humanos (Fuchs \& Bod 2006) e, em eqüinos como suplemento nutricional para atletas (Harris et al. 2006).

A glutamina pode ser administrada pelas vias parenteral ou oral e as doses encontradas na literatura são variáveis. No entanto, em humanos recomenda-se uma dose não superior a $25 \mathrm{mg} / \mathrm{kg}$ (Elia \& Lunn 1997). Para eqüinos atletas, a recomendação diária foi de 30 e $60 \mathrm{mg} / \mathrm{kg}$, por via oral (Harris et al. 2006). Não foi encontrada na literatura consultada referência sobre doses e vias de administração em eqüinos portadores de afecções intestinais.

O objetivo desse trabalho foi avaliar os possíveis efeitos da glutamina nas lesões de isquemia e reperfusão em modelos de distensão e isquemia venosa no jejuno de eqüinos.

\section{MATERIAL E MÉTODOS}

\section{Animais e anestesia}

Foram utilizados 12 eqüinos hígidos, sem raça definida, com idade de 4-12 anos, peso corporal de 297,4 $\pm 32,4 \mathrm{~kg}$, e escore entre 3 e 4 em escala de 1 a 5 (Spiers 1997). Os animais foram vermifugados e alimentados com feno à vontade nos 15 dias anteriores à cirurgia.

Após jejum de $12 \mathrm{~h}$, os animais foram pré-medicados com midazolam $(0,15 \mathrm{mg} / \mathrm{kg}$, IV) e éter gliceril guaiacol $(100 \mathrm{mg} / \mathrm{kg}$, IV) e entubados, induzidos e mantidos em plano anestésico por meio de anestesia inalatória com isofluorano. No transcirúrgico, a pressão arterial média foi mantida entre 70 e $100 \mathrm{~mm} \mathrm{Hg}$ por meio da infusão de dobutamina $(1-5 \mathrm{mcg} / \mathrm{kg} / \mathrm{min})$ diluída em solução de Ringer lactato, quando necessário. A concentração de $\mathrm{CO}_{2}$ ao final da expiração $\left(\mathrm{ETCO}_{2}\right)$ foi avaliada por capnografia e mantida entre 35 e $50 \mathrm{~mm} \mathrm{Hg}$ por respiração controlada.

O projeto CVZ 23704, protocolado com no 144/04, foi aprovado pelo Comitê de Ética em Experimentação Animal (CETEA/UFMG).

\section{Lesão experimental}

Através de celiotomia mediana foram demarcados dois segmentos de jejuno de $40 \mathrm{~cm}$ cada. O primeiro segmento, a um metro em sentido oral da prega íleo-cecal, foi submetido à isquemia venosa completa (IVC) e o segundo, situado a um metro de distância do primeiro em sentido oral, à distensão intraluminal (DIL).

Para instrumentar a IVC no jejuno, foram praticadas ligaduras murais nas extremidades do segmento experimental, utilizando-se drenos de látex tipo penrose $\mathrm{n} \div 3$ circundando externamente a parede intestinal, com o propósito de ocluir a irrigação e a drenagem mural e os vasos paralelos à borda mesentérica. Realizaram-se também ligaduras nos ramos das veias mesentéricas, utilizando-se drenos de penrose $\mathrm{n}$ ํ 1 . O suprimento sanguíneo oriundo da artéria mesentérica cranial mantido durante o período de isquemia e avaliada por meio de doppler ultrasônico.

A DIL do jejuno foi realizada por injeção intraluminal de solução salina ( $\mathrm{NaCl}$ 0,9\%) em um segmento isolado por ligaduras completas murais, como anteriormente descrito. A solução foi injetada via sonda uretral no 8, introduzida no lúmen através de enterotomia em segmento adjacente, chegando ao segmento isolado pela ligadura mural. A sonda foi acoplada externamente a um frasco de solução fisiológica e a um manômetro aneróide por meio de uma torneira de três vias. A pressão no lúmen intestinal foi mantida em $21 \mathrm{~cm}$ de água por todo o período de obstrução (Dabareiner et al. 2001).

Após a instrumentação, os segmentos experimentais foram mantidos obstruídos por duas horas, quando as ligaduras e sondas foram retiradas e os animais mantidos em anestesia durante as duas primeiras horas de reperfusão. Após esse tempo, a anestesia foi interrompida e a recuperação anestésica foi permitida.

Imediatamente ao final da anestesia, os animais foram medicados com butorfanol $(0,05 \mathrm{mg} / \mathrm{kg}$, IV) a cada quatro horas. Após $12 \mathrm{~h}$ de reperfusão, os animais foram sedados com xilazina $(1 \mathrm{mg} / \mathrm{kg}, \mathrm{IV})$ e acepromazina $(0,1 \mathrm{mg} / \mathrm{kg}, \mathrm{IV})$, e induzidos à anestesia geral com tiopental (3mg/kg, IV), procedendo-se, então, a eutanásia por infusão intravenosa de solução saturada de sulfato de magnésio.

\section{Tratamentos}

Os animais foram divididos aleatoriamente em Gl (controle) e Gll (glutamina ${ }^{8}$ ). Os tratamentos foram realizados $1 \mathrm{~h}$ após a

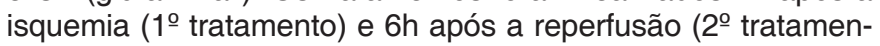
to) por via intravenosa (Quadro 1). A glutamina foi diluída em uma concentração a $2 \%$ em solução de ringer lactato aquecido a $37^{\circ} \mathrm{C}$ e a dose total utilizada foi de $50 \mathrm{mg} / \mathrm{kg}$. A taxa de infusão utilizada no GI foi a mesma utilizada no Gll sendo correspondente ao volume necessário para que a dose de glutamina cal-

\footnotetext{
${ }^{8}$ L-Glutamina (Galena Química e Farmacêutica Ltda, Campinas, SP).
} 


\section{Quadro 1. Grupos experimentais e metodologia de tratamento em 12 eqüinos submetidos à isquemia e reperfusão por distensão intraluminal e isquemia venosa de jejuno

\begin{tabular}{|c|c|c|}
\hline \multirow{2}{*}{$\begin{array}{c}\text { Grupo } \\
\text { experimental }\end{array}$} & \multicolumn{2}{|c|}{ Metodologia de tratamento } \\
\hline & $\begin{array}{l}1^{\circ} \text { Tratamento } \\
\text { (após } 1 \mathrm{~h} \text { de isquemia) }\end{array}$ & $\begin{array}{c}2^{\circ} \text { Tratamento } \\
\text { (após } 6 \mathrm{~h} \text { de reperfusão) }\end{array}$ \\
\hline Controle (Gl) & ão ringer lactato $12,5 \mathrm{~mL}$ & ão ringer lactato $12,5 \mathrm{~m}$ \\
\hline Glutamina (GII) & $\begin{array}{c}25 \mathrm{mg} / \mathrm{kg} \text { em solução de ringer lactato } \\
\text { a } 2 \% \text { em infusão de } 12,5 \mathrm{~mL} / \mathrm{kg} / \mathrm{h}\end{array}$ & $\begin{array}{c}25 \mathrm{mg} / \mathrm{kg} \text { em solução de ringer lactato } \\
\text { a } 2 \% \text { em infusão de } 12,5 \mathrm{~mL} / \mathrm{kg} / \mathrm{h}\end{array}$ \\
\hline
\end{tabular}

culada e preparada na concentração a $2 \%$ fosse administrada no período de $1 \mathrm{~h}$.

\section{Colheita, processamento e análise das amostras}

Foram colhidas amostras de tecido intestinal na borda antimesentérica por meio de secção transversal de todas as camadas antes e ao final do período de isquemia e após 2 e $12 \mathrm{~h}$ do período de reperfusão. A ferida foi suturada com categute 2-0, em padrão simples contínuo seguido de sutura invaginante em padrão Cushing. Após a eutanásia dos animais foram colhidas amostras do jejuno correspondentes a $12 \mathrm{~h}$ de reperfusão nos segmentos DIL e IVC e, em áreas distantes em média 1m dos segmentos experimentais, correspondendo ao segmento à distância da lesão, portanto, em um total de três amostras intestinais.

As amostras intestinais foram divididas em três fragmentos. O primeiro foi imediatamente fixado em solução de formalina tamponada a $10 \%$ e, posteriormente, processado segundo técnicas rotineiras de inclusão em parafina, cortadas em micrótomo a $5 \mathrm{~mm}$, coradas pela hematoxilina eosina para exame em microscópio de luz. O segundo fragmento foi fixado em solução de glutaraldeído tamponado e armazenado a $4^{\circ} \mathrm{C}$, para posterior processamento de rotina para microscopia eletrônica de varredura (MEV). O terceiro fragmento foi acondicionado em tubos criogênicos e imediatamente congelado por imersão em nitrogênio líquido para a realização das análises bioquímicas.

As análises histológicas foram realizadas por examinador sem o conhecimento da identificação do segmento avaliado, utilizando microscópio de luz em aumento de 40x. Foram utilizados parâmetros semi-quantitativos de escores para descamação epitelial, edema, hemorragia e infiltração de neutrófilos na mucosa, submucosa, muscular e serosa, segundo sistema adotado Rio Tinto (1999). Na serosa também foram utilizados escores para descamação de células mesoteliais, distensão de vasos linfáticos e deposição de fibrina, com base em método adotado por Dabareiner et al. (2001).

As amostras intestinais para a MEV foram preparadas mediante o corte de fragmentos de aproximadamente $16 \mathrm{~mm}^{2}$ para posterior tratamento com tetróxido de ósmio a $2 \%$ por duas horas e desidratação por passagens consecutivas de 15 minutos em concentrações crescentes de álcool etílico. Após secagem em $\mathrm{CO}_{2}$ sob pressão, por meio de Secador de Ponto Crítico, os fragmentos foram metalizados em metalizador de fluxo iônico por período de 70 segundos em potência de $70 \mathrm{~mA}$. A mucosa foi examinada em microscópico eletrônico de varredura, operando em $15 \mathrm{kV}$ em um aumento de 750 vezes. As amostras foram fotografadas e analisadas para avaliação de possíveis alterações morfológicas.

A partir das imagens obtidas pela MEV realizou-se a mensuração da área das vilosidades e do número de vilosidades por $\mathrm{mm}^{2}$ nos segmentos de jejuno submetidos à distensão intraluminal. Depois de digitalizadas, as imagens obtidas pela MEV foram avaliadas por meio de um software de processamento e análise de imagens de domínio público (Image J, National Institute of Mental Health, http://rsb.info.nih.gov/ij/). Após a calibração do cursor, ao se estabelecer a escala de número de pixels para $\mathrm{mcM}$, foram registrados o diâmetro e o perímetro do ápice da cada uma das vilosidades intestinais. $O$ valor de cada amostra foi considerado como o valor médio das vilosidades medidas. Para a contagem do número de vilosidades por $\mathrm{mm}^{2}$ foram considerados todos os ápices das vilosidades que apareciam por inteiro. Estabeleceu-se que seriam também incluídos nessa contagem todos os ápices incompletos presentes nas margens superior e esquerda, sendo desconsiderados aqueles situados nas margens inferior e direita.

A atividade de mieloperoxidase (MPO) foi avaliada nos fragmentos acondicionados em nitrogênio líquido. Após descongelamento, $100 \mathrm{mg}$ tecido intestinal foram triturados e homogeneizados em 1,9mL de solução tampão com pH 4,7 $(0,1 \mathrm{M}$ de $\mathrm{NaCl}$, $0,02 \mathrm{M}$ de $\mathrm{NaPO}_{4}, 0,015 \mathrm{M}$ de NaEDTA) e centrifugados a $260 \mathrm{x}$ $\mathrm{g}$ por $10 \mathrm{~min}$. Após a retirada do sobrenadante, foi procedida a lise hipotônica, com $1 \mathrm{~mL}$ de solução de $\mathrm{NaCl}$ a $0,2 \%$ por 30 segundos, seguida pela adição de solução com $1,6 \%$ de $\mathrm{NaCl}$ e $5 \%$ de glicose. Após nova centrifugação, foram realizadas ressuspensão e homogeneização com tampão de $\mathrm{NaPO}_{4}(\mathrm{pH}$ 5,4 ) contendo $0,5 \%$ de bromido de hexadecil-trimetil-amonia. Alíquotas de $1 \mathrm{~mL}$ dessa solução foram submetidas a três ciclos de congelamento em nitrogênio líquido. Após centrifugação por mais $10 \mathrm{~min}$. (10.000 x g), retirada do sobrenadante e ressuspensão em $1 \mathrm{~mL}$, determinou-se a atividade da MPO medindo-se a mudança na densidade ótica a 450nm usandose tetrametilbenzidina $(1,6 \mathrm{mM})$ e peróxido de hidrogênio $(0,5 \mathrm{mM})$.

\section{Análise dos dados}

Foram realizadas comparações entre os tempos de cada grupo e entre os grupos. As avaliações estatísticas paramétricas foram feitas através de análise de variância em blocos ao acaso e submetidas ao teste de Student-Newman-Keuls para comparação tempos de um mesmo grupo. O teste t de Student foi utilizado para comparação de grupos em cada tempo. Os dados não paramétricos foram analisados segundo o teste de Friedman para tempos em um mesmo grupo e o teste MannWhitney para grupos em um mesmo tempo (Sampaio, 1998) com nível de significância de P£0,05.

\section{RESULTADOS}

Pela avaliação histopatológica a lesão de mucosa do jejuno foi ausente nos segmentos DIL, ocorrendo apenas nos segmentos IVC. Nesses segmentos, a lesão iniciouse em ambos os grupos após $2 \mathrm{~h}$ de isquemia, acentuando-se às $2 \mathrm{~h}$ de reperfusão com atenuação às $12 \mathrm{~h}$ (Qua- 
Quadro 2. Valores médios e erro padrão para os escores de lesão de mucosa e edema de submucosa do jejuno de eqüinos do grupo controle (GI) e tratados com glutamina (GII) a $2 \%$ (50mg/ kg, IV) submetidos a modelo de isquemia e reperfusão por isquemia venosa nos tempos basal, $2 \mathrm{~h}$ de isquemia e 2 e 12h de reperfusão e segmento distante à lesão após $12 \mathrm{~h}$ de reperfusão

\begin{tabular}{|c|c|c|c|c|c|c|c|c|c|c|}
\hline & \multicolumn{5}{|c|}{ Grupo Controle (GI) } & \multicolumn{5}{|c|}{ Grupo Glutamina (GII) } \\
\hline & \multicolumn{2}{|r|}{ Isquemia } & \multicolumn{2}{|c|}{ Reperfusão } & \multirow{2}{*}{$\begin{array}{c}\text { Segmento } \\
\text { distante }\end{array}$} & \multicolumn{2}{|r|}{ Isquemia } & \multicolumn{2}{|c|}{ Reperfusão } & \multirow{2}{*}{$\begin{array}{c}\text { Segmento } \\
\text { distante }\end{array}$} \\
\hline & Basal & $2 \mathrm{~h}$ & $2 \mathrm{~h}$ & $12 \mathrm{~h}$ & & Basal & $2 \mathrm{~h}$ & $2 \mathrm{~h}$ & $12 \mathrm{~h}$ & \\
\hline Lesão & $0,0 a A$ & $3,3 a A$ & $4,2 \mathrm{bA}$ & $3,9 b A$ & $1,0 \mathrm{aA}$ & $0,0 \mathrm{aA}$ & $3,3 b A$ & $3,9 b A$ & $2,3 a A$ & $0,5 \mathrm{aA}$ \\
\hline Mucosa & 0,0 & 0,3 & 0,2 & 0,5 & 0,3 & 0,0 & 0,5 & 0,4 & 0,6 & 0,2 \\
\hline Edema & $0,0 a A$ & $2,4 \mathrm{bA}$ & $2,1 \mathrm{aA}$ & $2,0 \mathrm{aA}$ & $0,2 \mathrm{aA}$ & $0,0 \mathrm{aA}$ & $2,8 \mathrm{bA}$ & $2,1 \mathrm{aA}$ & $1,9 a A$ & $0,0 \mathrm{aA}$ \\
\hline Submucosa & 0,0 & 0,4 & 0,6 & 0,6 & 0,2 & 0,0 & 0,3 & 0,5 & 0,1 & 0,0 \\
\hline
\end{tabular}

Médias seguidas de mesma letra minúscula não diferem entre si em relação ao tempo dentro do mesmo grupo; médias seguidas de mesma letra maiúsculas não diferem entre si entre grupos.

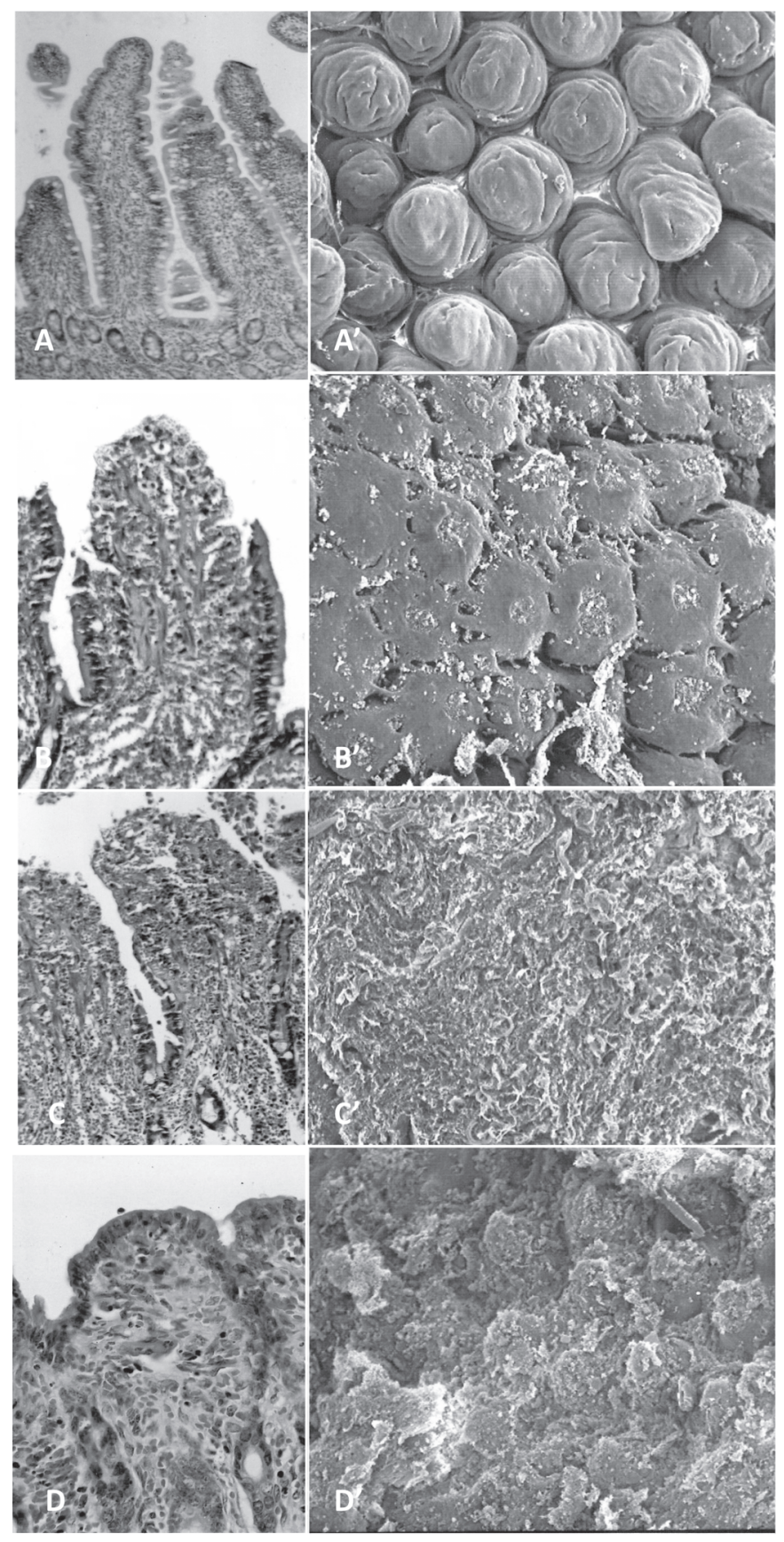

dro 2). A hemorragia foi mais presente nos segmentos IVC, sendo significativa após $2 \mathrm{~h}$ de isquemia na mucosa de ambos os grupos. Nos segmentos DIL, a hemorragia limitou-se a serosa, porém sem aumento significativo. $\mathrm{O}$ edema foi significativo em ambos os grupos apenas na submucosa do segmento IVC após $2 \mathrm{~h}$ de isquemia, diminuindo nos tempos subseqüentes (Quadro 2). O infiltrado de neutrófilos foi mais acentuado na serosa dos segmentos IVC e DIL sendo significativo apenas no grupo controle após $12 \mathrm{~h}$ de reperfusão, porém sem diferença entre grupos. O desprendimento de células mesenquimais ocorreu em ambos os grupos, tanto nos segmentos DIL quanto IVC, sendo significativo apenas às $2 \mathrm{~h}$ de isquemia do grupo GII. Em nenhuma das variáveis analisadas houve diferença significativa entre grupos.

A microscopia eletrônica de varredura (MEV) permitiu avaliar de forma detalhada as lesões de mucosa observadas na histopatologia (Fig.1). Em todas as amostras dos segmentos DIL as vilosidades observadas à MEV estavam íntegras, correspondendo ao observado na histopatologia. As imagens obtidas pela MEV também possibilitaram a mensuração da área e do número de vilosidades por $\mathrm{mm}^{2}$. A área das vilosidades foi maior e o número de vilosidades por $\mathrm{mm}^{2}$ foi menor em ambos os grupos após $12 \mathrm{~h}$ de reperfusão, porém sem diferença significativa entre os grupos.

A atividade de MPO teve aumento significativo em ambos os grupos após 2 e $12 \mathrm{~h}$ de reperfusão nos segmentos DIL e IVC, respectivamente. Nos segmentos de jejuno distantes à lesão, o aumento foi significativo em ambos os grupos. No entanto, não houve diferença signi-

Fig.1. Progressão das lesões de mucosa do tempo 0 (2 h antes da isquemia) até $12 \mathrm{~h}$ de reperfusão pela microscopia óptica (A, B, C, D, aumento de 40x) e microscopia eletrônica de varredura (A',B',C',D', aumento de 150x). Escore 0 (tempo $0)$ : mucosa íntegra (A e $\left.\mathbf{A}^{\prime}\right)$; Escore 3 (2h de isquemia): exposição da lâmina própria até a metade da extensão das vilosidades (B e B'); Escore 4 (2 h de reperfusão): exposição da lâmina própria até a base das vilosidades (C e C'); Vilosidades curtas, mas com recobrimento de células epiteliais (12 h de reperfusão) (D e D') (Eqüino do Grupo Controle). 


\begin{tabular}{|c|c|c|c|c|c|c|c|c|c|c|}
\hline \multicolumn{11}{|c|}{ Jejuno Isquemia Venosa (IVC) } \\
\hline \multicolumn{5}{|c|}{ Controle } & \multicolumn{5}{|c|}{ Glutamina } & \\
\hline T0 & 12 & $\mathrm{R} 2$ & $\mathrm{R} 12$ & $\mathrm{R} 12 \mathrm{~L}$ & T0 & 12 & $\mathrm{R} 2$ & $\mathrm{R} 12$ & $\mathrm{R} 12 \mathrm{~L}$ & \\
\hline$M^{*}$ & $0,005 a A$ & $0,062 b A$ & $0,098 \mathrm{bA}$ & $0,431 \mathrm{bA}$ & $0,467 \mathrm{bA}$ & $0,003 a A$ & $0,071 \mathrm{bA}$ & $0,054 \mathrm{bA}$ & $0,613 b A$ & $0,305 \mathrm{Ba}$ \\
\hline SD & 0,0097 & 0,0574 & $\begin{array}{c}0,1839 \\
\text { Jejuno }\end{array}$ & $\begin{array}{l}0,4667 \\
\text { o Distensã }\end{array}$ & $\begin{array}{c}0,4683 \\
\text { Intralumine }\end{array}$ & $\begin{array}{l}0,0023 \\
\text { al (Dil) }\end{array}$ & 0,126 & 0,0832 & 0,3291 & 0,2581 \\
\hline $\begin{array}{l}\mathrm{M}^{*} \\
S D\end{array}$ & $\begin{array}{c}0,005 \mathrm{aA} \\
0,0097\end{array}$ & $\begin{array}{c}0,042 \mathrm{aA} \\
0,0285\end{array}$ & $\begin{array}{c}0,739 \mathrm{bA} \\
0,3857\end{array}$ & $\begin{array}{c}0,703 \mathrm{bA} \\
0,3065\end{array}$ & $\begin{array}{c}0,466 \mathrm{bA} \\
0,4683\end{array}$ & $\begin{array}{c}0,003 \mathrm{aA} \\
0,0023\end{array}$ & $\begin{array}{c}0,051 \mathrm{aA} \\
0,0417\end{array}$ & $\begin{array}{c}0,819 \mathrm{bA} \\
0,4157\end{array}$ & $\begin{array}{c}0,769 \mathrm{bA} \\
0,5082\end{array}$ & $\begin{array}{c}0,305 \mathrm{aA} \\
0,2581\end{array}$ \\
\hline
\end{tabular}

*Médias seguidas entre si pela mesma letra minúscula não diferem entre si no mesmo tempo e seguidas entre si pela mesma letra maiúscula não diferem entre si entre grupos.

ficativa entre grupos em nenhum dos segmentos e tempos avaliados.

\section{DISCUSSÃO}

Nos eqüinos desse experimento, houve um agravamento das lesões de mucosa iniciadas na isquemia durante a reperfusão, o que ocorre devido ao mecanismo fisiopatológico desenvolvido ao longo desses eventos. Durante a isquemia, a redução da oferta de oxigênio bloqueia a produção de ATP na cadeia fosforilativa, o que inviabiliza a homeostase celular, induz à necrose tecidual e ativa a enzima xantina-oxidase. Com o retorno de oxigênio durante a fase de reperfusão, essa enzima ocasiona aumento da concentração de radicais livres de oxigênio (RLO), o que agrava a lesão previamente iniciada na fase de isquemia (Rio Tinto 1999).

O edema na submucosa observado nos eqüinos desse experimento nos segmentos IVC na fase de isquemia pode ter sido decorrente de um aumento da pressão hidrostática local. Durante a isquemia, o fluxo venoso foi obstruído concomitante com a manutenção do fluxo arterial nesse segmento, o que promove um aumento da pressão hidrostática nas vênulas e um extravasamento de líquido para o interstício, colaborando para o edema. Com a reperfusão, o restabelecimento do fluxo venoso facilitou o fluxo de líquido acumulado no interstício para as vênulas, o que colaborou para a diminuição do edema nos tempos subseqüentes em ambos os grupos. Um padrão semelhante foi observado por Rio Tinto (1999) utilizando o mesmo modelo experimental em eqüinos.

$\mathrm{O}$ aumento da área das vilosidades e a diminuição do número de vilosidades por $\mathrm{mm}^{2}$ observado no presente experimento às $12 \mathrm{~h}$ de reperfusão em ambos os grupos não foi ocasionado por edema ou inflamação, uma vez que não foram visualizados na microscopia óptica alterações compatíveis. Esses resultados, no entanto, podem ter sido decorrentes de um achatamento das vilosidades devido à distensão intraluminal pelo acúmulo de gás observado durante a abertura abdominal para a colheita de amostras após a eutanásia dos animais. $O$ acúmulo de gás pode ocorrer devido à atonia ocasionada por lesão intestinal ou fermentação bacteriana (Moore 1992), ambas passíveis de ter ocorrido nos eqüinos desse experimento.

A MPO é uma enzima decorrente de neutrófilos ativados, sendo um recurso para a medida quantitativa indireta da presença dessas células e, portanto, de inflamação. Normalmente, seu aumento ocorre concomitante com a presença de infiltrado neutrofílico (Faleiros et al., 2008). No entanto, nos eqüinos desse experimento, o aumento de MPO não foi acompanhado por aumento do infiltrado de neutrófilos. Nos segmentos DIL após $2 \mathrm{~h}$ de reperfusão essas células não foram visualizadas à microscopia óptica. A justificativa para tal achado pode ser pelo fato da MPO não ser uma enzima específica de neutrófilos, podendo ser decorrente da atividade de eosinófilos e monócitos (Krawisz et al. 1984). No intestino de eqüinos há abundância de eosinófilos em condições normais e essas células podem ser as principais constituintes do infiltrado inflamatório nas primeiras $24 \mathrm{~h}$ após injúria intestinal, juntamente com os neutrófilos, contribuindo para aumentar a concentração de MPO (McConnico et al. 1999). Assim sendo, nos eqüinos desse experimento, acredita-se que o aumento da MPO possa ter sido decorrente predominantemente de outras células que não os neutrófilos.

O aumento da produção de RLO em situações de I/R intestinal ocorre concomitante à diminuição dos mecanismos de neutralização desses radicais (Rio Tinto 1999). A glutamina é o principal precursor da glutationa, um agente antioxidante importante, sendo responsável pela manutenção de seus níveis durante o estresse oxidativo, situação na qual pode ocorrer sua depleção (AmoresSanchéz \& Medina 1999, Jia et al. 2006). Em eqüinos submetidos a I/R, o tratamento com glutationa atenuou 0 infiltrado de polimorfonucleares e diminuiu o edema de submucosa do jejuno (Abreu 2003).

A ação da glutamina na neutralização e diminuição da formação de RLO têm o potencial de proteger e manter a integridade do epitélio intestinal em situações de l/R, evitando ou atenuando a lesão da mucosa. Adicionalmente, esse nutriente também estimula a proliferação de enterócitos, o que auxilia na regeneração do epitélio intestinal após o estabelecimento da lesão (Blikslager \& Roberts 1997, Thomas \& Balasubramanian 2005). No entanto, no 
presente experimento, nenhum desses efeitos foi obtido o que pode ter sido decorrente da metodologia utilizada, a qual diferiu da encontrada na literatura consultada.

Em eqüinos, a glutamina é utilizada como suplemento na dieta de atletas, apesar de reconhecer-se que seus níveis estão diminuídos em casos de infecção na espécie (Harris et al. 2006). Nos eqüinos do presente experimento, optou-se pela administração intravenosa, uma vez que a absorção intestinal é limitada em casos de lesões. Em humanos com afecções intestinais e com ingestão alimentar limitada, a corrente circulatória é a fonte mais importante de glutamina para o intestino (Elia \& Lunn 1997).

Nesse trabalho, utilizou-se a dose de $50 \mathrm{mg} / \mathrm{kg}$, a qual está entre os valores utilizados na suplementação nutricional de eqüinos atletas (Harris et al. 2006). Nessa forma de suplementação, as concentrações plasmáticas de glutamina elevam-se $1 \mathrm{~h}$ após a ingestão, sem aumentos subseqüentes da amônia plasmática, diminuindo em até 8h. Assim, optou-se pelo fracionamento da dose em dois momentos, com intervalo de seis horas entre ambos, com o objetivo de se manterem constantes as concentrações plasmáticas de glutamina. No entanto, a dose utilizada não atenuou as lesões decorrentes de I/R, devendo-se considerar doses maiores para tal finalidade. $\mathrm{Na}$ avaliação da diminuição da resposta inflamatória pós-parto em bovinos leiteiros, a glutamina foi utilizada nas doses de 159 e 319mg/ $\mathrm{kg}$, tendo ambas igual eficácia (Jafari et al. 2006).

Além da dose utilizada, outro fator que pode ter colaborado para o insucesso do tratamento foi o modo de administração da glutamina, a qual diferiu da metodologia utilizada nos experimentos de isquemia e reperfusão na literatura consultada. Nesses experimentos, a administração foi realizada de 3-72 $\mathrm{h}$ antes da isquemia em infusão intravenosa contínua (Harward et al. 1994, Jia et al. 2006, Szijártó et al. 2007). Entretanto, é fundamental ressaltar que essa utilização é praticamente inviável em casos de eqüinos com cólica, uma vez que seria necessário prever sua ocorrência para iniciar o tratamento com a glutamina. No entanto, é pertinente considerar que a metodologia utilizada nesse experimento pudesse ter sido a de infusão contínua, mesmo após o estabelecimento da isquemia e sugere-se que essa metodologia seja utilizada, juntamente com doses superiores, em experimentos futuros com eqüinos submetidos ao mesmo modelo experimental.

A mucosa intestinal utiliza a maior parte da glutamina circulante, aumentando grandemente sua demanda em situações de estresse oxidativo ou inflamatório (Fuchs \& Bode 2006). Porém, como o intestino é incapaz de armazenar esse nutriente, depende das quantidades presentes na corrente circulatória ou na dieta. Adicionalmente, a glutamina é um dos constituintes do muco, além de atuar no co-transporte de líquidos pelo intestino (Elia \& Lunn 1997), o que a torna uma substância com elevado potencial benéfico no tratamento de lesões intestinais decorrentes isquemia e reperfusão. Assim, mesmo que os re- sultados obtidos nesse experimento tenham sido opostos aos encontrados na literatura e na hipótese desse trabaIho, considera-se que a glutamina não deve ser descartada como um possível recurso terapêutico em eqüinos portadores de isquemia e reperfusão intestinal.

\section{CONCLUSÃO}

O tratamento com glutamina na dose de $50 \mathrm{mg} / \mathrm{kg}$ por via intravenosa não atenuou as lesões de isquemia e reperfusão do jejuno em eqüinos.

Agradecimentos.- À Fundação de Amparo à Pesquisa de Minas Gerais (FAPEMIG, Proc. CAG-237/04).

\section{REFERÊNCIAS}

Abreu J.M.G. 2003. Apoptose e efeitos da pentoxifilina, da glutationa e do ozônio no jejuno de eqüinos sob isquemia, congestão e reperfusão. Tese de Doutorado em Ciência Animal, Universidade Federal de Minas Gerais, Belo Horizonte. 91p.

Amores-Sánchez M.I. \& Medina M.A. 1999. Glutamine, as a precursor of glutathione, and oxidative stress. Mol. Gen. Metab. 67:100-105.

Blikslager A.T. \& Roberts M.C. 1997. Mechanisms of intestinal mucosal repair. J. Am. Vet. Med. Assoc. 211:1437-1438.

Dabareiner R.M., Sullins K.E., White N.A. \& Snyder J.R. 2001. Serosal injury in the equine jejunum and ascending colon after ischemiareperfusion or intraluminal distention and decompression. Vet. Surg. 30:114-125

Elia M. \& Lunn P.G. 1997. The use of glutamine in the treatment of gastrointestinal disorders in man. Nutrition 13:743-747.

Flaherty J.T. \& Weisfeldt M.L. 1988. Reperfusion injury. Free Rad. Biol. Med. 5:409-419.

Faleiros R.R., Macoris D.G., Alves G.E.S., Souza D.G., Teixeira M.M. \& Moore R.M. 2008. Local and remote lesions in horses subjected to small colon distension and decompression. Can. J. Vet. Res. 72:68-76.

Forsyth S.F. \& Guilford W.G. 1995. Ischemia-reperfusion injury: A small animal perspective. Brit. Vet. J. 151:281-198.

Fuchs B.C. \& Bode B.P. 2006. Stressing out over survival: Glutamine as an apoptotic modulator. J. Surg. Res. 131:26-40.

Harris R.C., Harris P.A., Routledge N.B.H., Naylor J.R.J. \& Wilson A.M. 2006. Plasma glutamine concentrations in the horse following feeding and oral glutamine supplementation. Equine Vet. J. 36 (Suppl.):637642.

Harward T.R.S., Douglas C., Souba W.W., Klingman N. \& Seeger J.M. 1994. Glutamine preserves gut glutathione levels during intestinal ischemia/reperfusion. J. Surg. Res. 56:351-355.

Jafari A., Emmanuel D.G.V., Christopherson R.J., Thompson J.R., Murdoch G.K., Woodward J., Field C.J. \& Ametaj B.N. 2006. Parenteral administration of glutamine modulates acute phase response in postparturient dairy cows. J. Dairy Sci. 89:4660-4668.

Jia C.J., Dai C.L., Zang X., Cui K., Xu F. \& Xu Y.Q. 2006. Alanyl-glutamine dipeptide inhibits Hepatic ischemia-reperfusion injury in rats. World J. Gastroenterol. 12:1373-1378.

Krawisz J.E., Sharon P. \& Stenson W.F. 1984. Quantitative assay for acute intestinal inflammation based on myeloperoxidase activity. Gastroenterology 87:1344-1350.

Lopes-Paulo F. 2005. Efeitos da glutamina sobre a parede intestinal e sua aplicabilidade potencial em coloproctologia. Revta Bras. Coloproct. 25:75-78.

McConnico R.S., Weinstock D., Poston M.E. \& Roberts M.C. 1999. Myeloperoxidase activity of the large intestine in an equine model of acute colitis. Am. J. Vet. Res. 60:807-813. 
Moore R.M. 1992. Pathophysiology of intestinal ischemia and endotoxemia. Equine Prac. 14:13-15.

Murphy C.G., Chen G., Winter D.C. \& Hayes-Bouchier D.J. 2007. Glutamine preconditioning protects against tour-niquet-induced and distant organ injury in a rodent ischemia-reperfusion model. Acta Orthopaedica 78:559-566.

Peng X., Yan H., You Z., Wang P. \& Wang S. 2004. Effects of enteral supplementation with glutamine granules on intestinal mucosal barrier function in severe burned patients. Burns 30:135-139.

Rio Tinto J.J. 1999. Lesões de isquemia e reperfusão experimentais no jejuno de eqüinos: tratamento com succinato sódico de hidrocortisona. Dissertação de Mestrado em Clínica e Cirurgia Veterinárias, Universidade Federal de Minas Gerais. Belo Horizonte. 82p.

Sampaio I.B.M. 1998. Delineamento em blocos ao acaso, p.50-60. In: (Ed.), Estatística Aplicada à Experimentação Animal. Editora FEPMZV, Belo Horizonte.
Speirs V.C. 1997. The alimentary tract, p.261-298. In: Examination of Horses. W.B. Saunders, Philadelphia.

(Ed.), Clinical

Szijártó A., Hahn O., Batmunkh E., Stangl R., Kiss A., Lotz G., Schaff Z., Váli L., Blázovics A., Geró D., Szabó C., Kupcsulik P. \& Harsányi L. 2007. Short-term alanyl-glutamine dipeptide pretreatment in liver ischemia-reperfusion model: Effects on microcirculation and antioxidant status in rats. Clin. Nutr. 26:640-648.

Thomas S., Prabhu R. \& Balasubramanian K.A. 2005. Surgical manipulation of the intestnie and distant organ damage: Protection by oral glutamine supplementation. Surgery 137:48-55.

Wasa M., Soh H., Shimizu Y. \& Fukuzawa M. 2005. Glutamine stimulates amino acid transport during ischemia-reperfusion in human intestinal epithelial cells. J. Surg. Res. 123:75-81.

White N.A. 1990. Epidemiology and etiology of colic, p.49-64. In: (Ed.), The Equine Acute Abdomen. Lea and Febiger, Philadelphia. White N.A. 1992. Conditions of the small intestine. Equine Pract. 14:16-21. 